\title{
A dynamic programming approach for pricing options embedded in bonds
}

\author{
Hatem Ben-Ameur ${ }^{\mathrm{a}}$, Michèle Breton ${ }^{\mathrm{a}, *}$, Lotfi Karoui ${ }^{\mathrm{b}}$, \\ Pierre L'Ecuyer ${ }^{\mathrm{c}}$ \\ ${ }^{\mathrm{a}}$ GERAD, CREF, and HEC Montréal, 3000, chemin de la Côte-Sainte-Catherine, Montréal (Québec), \\ Canada H3T 2 A7 \\ ${ }^{\mathrm{b}}$ McGill University, Montréal, Canada \\ ${ }^{\mathrm{c}}$ GERAD and Université de Montréal, Montréal, Canada
}

Received 8 December 2004; accepted 21 June 2006

Available online 1 September 2006

\begin{abstract}
We propose a dynamic programming (DP) approach for pricing options embedded in bonds, the focus being on call and put options with advance notice. An efficient procedure is developed for the cases where the interest-rate process follows the Vasicek, Cox-IngersollRoss (CIR), or generalized Vasicek models. Our DP methodology uses the exact joint distribution of the interest rate and integrated interest rate at a future date, conditional on the current value of the interest rate. We provide numerical illustrations, for the Vasicek and CIR models, comparing our DP method with finite-difference methods. Our procedure compares quite favorably in terms of both efficiency and accuracy. An important advantage of the our DP approach is that it can be applied to more general models calibrated to capture the term structure of interest rates (e.g., the generalized Vasicek model).
\end{abstract}

(C) 2006 Elsevier B.V. All rights reserved.

JEL classification: $\mathrm{C} 63 ; \mathrm{G} 13$

Keywords: Option pricing; Bond pricing; Dynamic programming; Options embedded in bonds

\footnotetext{
*Corresponding author. Tel.: + 15143406480 ; fax: + 15143405634 .

E-mail address: michele.breton@hec.ca (M. Breton).
} 


\section{Introduction}

A bond is a contract that pays its holder a known amount, the principal, at a known future date, called the maturity. The bond may also pay periodically to its holder fixed cash dividends, called the coupons. When it gives no dividend, it is known as a zero-coupon bond. A bond can be interpreted as a loan with a known principal and interest payments equal to the coupons (if any). The borrower is the issuer of the bond and the lender, i.e., the holder of the bond, is the investor.

Several bonds contain one or several options coming in various flavors. The call option gives the issuer the right to purchase back its debt for a known amount, the call price, during a specified period within the bond's life. Several government bonds contain a call feature (see Bliss and Ronn, 1995 for the history of callable US Treasury bonds from 1917). The put option gives the investor the right to return the bond to the issuer for a known amount, the put price, during a specified period within the bond's life. These options are an integral part of a bond, and cannot be traded alone, as is the case for call and put options on stocks (for example). They are said to be embedded in the bond. In general, they are of the American-type and, thus, allow for early exercising, so that the bond with its embedded options can be interpreted as an American-style interest-rate derivative. This paper focuses on call and put options embedded in bonds with advance notice, that is, options with exercise decisions prior to exercise benefits.

As it is often the case in practice, we assume that exercise of the call and put options is limited to the coupon dates posterior to a known protection period and that there is a notice period of fixed duration $\Delta t$. Thus, consider a coupon date $t_{m}$ where the exercise is possible, and let $C_{m}$ and $P_{m}$ be the call and put prices at $t_{m}$. The decision to exercise or not by the issuer and the investor must be taken at $t_{m}-\Delta t$. If the issuer calls back the bond at $t_{m}-\Delta t$, he pays $C_{m}$ to the investor at $t_{m}$, and, similarly, if the investor puts the bond at $t_{m}-\Delta t$, he receives $P_{m}$ from the issuer at $t_{m}$. If no option is exercised at $t_{m}$, by the no-arbitrage principle of asset-pricing (Elliott and Kopp, 1999), the value of the bond is equal to the expected value of the bond at the next decision date, discounted at the interest rate. This expectation is taken under the so-called risk-neutral probability measure, where the uncertainty lies in the future trajectory of the (risk-free short-term) interest rate.

There are no analytical formulas for valuing American derivatives, even under very simplified assumptions. Numerical methods, essentially trees and finite differences, are usually used for pricing. Recall that trees are numerical representations of discrete-time and finite-space models and finite differences are numerical solution methods for partial differential equations. The pricing of American financial derivatives can also be formulated as a Markov decision process, that is, a stochastic dynamic programming (DP) problem, as pointed out by Barraquand and Martineau (1995). Here the DP value function, that is, the value of the bond with its embedded options, is a function of the current time and of the current interest rate, namely the state variable. This value function verifies a DP recurrence via the no-arbitrage principle of asset pricing, the solution of which yields both the bond value and the 
optimal exercise strategies of its embedded options at all time during the bond's life. For an extensive coverage of stochastic DP, see Bertsekas (1995).

The pricing of options embedded in bonds can be traced back to Brennan and Schwartz (1977). They used a standard finite-difference approach, which is however known to be instable in this context. Much later, Hull and White (1990a) proposed trinomial trees in the context of generalized versions with time-dependent parameters for the state process. Trees crudely approximate the dynamics of the underlying asset and convergence is not easy to attain. Büttler and Waldvogel (1996) (indicated here by BW) suggested that finite differences could not be used to price callable bonds with advance notice because of discontinuities at notice dates. They proposed an approach based on Green's functions, which turns out to be a DP procedure combined with finite elements, where the transition parameters are obtained by numerical integration. Recently, d'Halluin et al. (2001) (which we indicate by DFVL) were able to stabilize the finite-difference approach via flux limiters and timestepping. Using a Crank-Nicholson time weighing, they compared their results with those of BW, concluding that their method showed better accuracy than other methods. Here, we propose a DP approach where the transition parameters are derived in closed-form. As BW and DFVL, we use the Vasicek and CIR specification of the interest rate dynamics for our numerical experiments. Results are compared to those of BW and DFVL and show the efficiency and robustness of our DP method, with an accuracy comparable to DFVL and superior to BW. An interesting advantage of our approach is that it can be applied to generalized models calibrated to capture the term structure of interest rates, such as the ones proposed by Hull and White (1990a).

The rest of the paper is organized as follows. Section 2 presents a short review of the interest rate dynamics that have been proposed in the literature. Section 3 presents the model, the general DP formulation and the approximation procedure. Section 4 derives the exact joint distribution of the interest rate and integrated interest rate at a future date, conditional on the current value of the interest rate, for the Vasicek, CIR, and generalized Vasicek models. These joint distributions are used to obtain closed-form formulas for some constants used in our DP procedure. This turns out to be a key ingredient for its efficiency. Section 5 reports our numerical experimentations for the Vasicek and CIR models. Section 6 contains a conclusion. Appendix A contains some auxiliary results and proofs.

\section{Dynamics of the interest rate}

As pointed out by Chan et al. (1992), most of the alternative dynamics for the interest rate are described by the general stochastic differential equation

$$
\mathrm{d} R(t)=\kappa(\bar{r}-R(t)) \mathrm{d} t+\sigma R(t)^{\theta} \mathrm{d} B(t) \text { for } 0 \leqslant t \leqslant T,
$$

where $\{B(t), t \geqslant 0\}$ is a standard Brownian motion whereas $\kappa$ (the reverting rate), $\bar{r}$ (the reverting level), $\sigma$ (the volatility), and $\theta$, are real-valued parameters. Various versions of (1) found in the literature are listed in the table below. Blank entries 
indicate that the corresponding parameters can take arbitrary values. On the last line, $\bar{r}(t)$ represents a deterministic function of $t$.

Interest-rate models

\begin{tabular}{lccc}
\hline Model & $\bar{r}$ & $\kappa$ & $\theta$ \\
\hline 1. Vasicek (1977) & & 0 & 0 \\
2. Brennan-Schwartz (1977) & & & 1 \\
3. Brennan-Schwartz (1980) & & \\
4. Marsh-Rosenfeld (1983) & & \\
5. Cox-Ingersoll-Ross (1985a, b) & $\bar{r}(t)$ & & $1 / 2$ \\
6. Hull-White (1990a) & & 0 or $1 / 2$ \\
\hline
\end{tabular}

Vasicek (1977) used a mean-reverting Ornstein-Uhlenbeck process. This model gives nice distributional results and closed-form solutions for zero-coupon bonds and for several European-style interest-rate derivatives. But it has the undesirable property of allowing negative interest rates, although with very low probabilities. Several authors took advantage of its properties to price various interest-rate derivatives, often in closed-form. Examples include Jamshidian (1989) and Rabinovitch (1989).

Brennan and Schwartz (1977, 1980) were pioneers on the modeling of options embedded in bonds. They let the interest rate move as a geometric Brownian motion without a drift to price the call and put options (Model 2) and as a mean-reverting proportional process to price the conversion option (Model 3). Model 2 was also used by Dothan (1978) to price bonds in closed-form, and Model 3 by Courtadon (1982) to price several European as well as American options on bonds. Notice that Model 2 is a special case of Model 3 and that the latter includes the geometric Brownian motion of Black and Scholes (1973).

Model 4 corresponds to the so-called constant-elasticity-of-variance process (Cox, 1996). It was considered by Marsh and Rosenfeld (1983), among others, as an alternative process for the interest rate.

Cox et al. (1985a,b) (CIR) used the mean-reverting square-root process to handle the interest rate movements. This model is extensible to several factors, ensures strictly positive interest rates, and gives closed-form solutions for zero-coupon bonds and for some European-style interest-rate derivatives. Several authors used the CIR model to price various interest-rate derivatives (e.g., Richard, 1978; Ananthanarayanan and Schwartz, 1980; Schaefer and Schwartz, 1984). Notice that the Vasicek and the CIR models are special cases of the so-called single-factor affine term structure models. Duffie and Kan (1996) provide a characterization of multifactor affine term structure models. For the models described by (1), matching all theoretical bond values with their market counterparts is unfeasible because this gives much more equations than the number of parameters to estimate. A remedy, proposed by Hull and White (1990a), is to consider time-dependent parameters to better adjust the model to the observed yield curves. This leads to the generalized 
Vasicek and CIR models. Hull and White (1990b, 1993, 1994a,b, 1996) interpreted the finite-difference method as a trinomial tree, and priced several interest-rate derivatives within their extended models. See also the note by Carverhill (1995) and the response by Hull and White (1995) for a discussion about the performance of these models.

\section{A dynamic programming approach}

In this section, we first provide a precise formulation of the model considered for a bond with its embedded call and put options. We then develop the DP equations and an approximation procedure to solve them. The pricing is made under a standard noarbitrage assumption.

\subsection{Model and formulation}

Let $t_{0}, \ldots, t_{n}$ be a sequence of dates, where $t_{0}=0$ is the initial time, $t_{1}, \ldots, t_{n-1}$ are the coupon dates, and $t_{n}=T$ is the maturity date of the bond, i.e., the time at which the principal and the last coupon are due. The principal is scaled to 1 and the corresponding coupon is denoted by $c$. The periods $t_{m+1}-t_{m}$, for $m=1, \ldots, n$, are equal except perhaps for the the first one, $t_{1}-t_{0}$, which can be different. Assume also that the exercise decisions of the call and put options are made at times $\tau_{m}=t_{m}-\Delta t>t_{m-1}$, for $m=n^{*}, \ldots, n$. The benefits of an exercise decision at $\tau_{m}$ are obtained at the coupon date $t_{m}$. The lag $\Delta t=t_{m}-\tau_{m}$ is the notice period and the time increment $\tau_{n^{*}}-t_{0}$ is the protection period (against early exercising). All these dates $t_{m}$, and the values of $\Delta t$ and $n^{*}$, are known in advance.

Let $C_{m}$ and $P_{m}$ be the call and put prices at $t_{m}$, for $m=n^{*}, \ldots, n$, respectively. Thus, if the issuer calls back the bond at $\tau_{m}$, he pays $C_{m}$ to the investor at $t_{m}$, and, similarly, if the investor puts the bond at $\tau_{m}$, he receives $P_{m}$ from the issuer at $t_{m}$. We assume that $0 \leqslant P_{m} \leqslant C_{m}$, as is usual in practice, and that $C_{n}=P_{n}=1$.

Let $E^{Q}[\cdot \mid R(\tau)=r]$ represent the expectation operator, conditional on the interest rate at time $\tau$, under the so-called risk-neutral probability measure $Q$. Specific models for the dynamics of the interest-rate process under $Q$ will be considered in Sections 4 and Appendix A. For $\delta \geqslant 0$, define

$$
\rho(r, \tau, \delta)=E^{Q}\left[\exp \left(-\int_{\tau}^{\tau+\delta} R(t) \mathrm{d} t\right) \mid R(\tau)=r\right],
$$

which represents the discount factor over the period $[\tau, \tau+\delta]$ when $R(\tau)=r$. Equivalently, this is the price at $\tau$ of a zero-coupon bond with a principal scaled to 1 and a maturity date $\tau+\delta$. Let $v(t, r)$ denote the value of the bond with its embedded options at time $t$ if $R(t)=r$ and assuming that both parties always make their decisions in an optimal way, i.e., each one maximizes its total expected discounted revenue. 
We adopt the convention that $\tau_{n+1}=t_{n}=T$ and $\tau_{m}=t_{0}$ for $m=0, \ldots, n^{*}-1$, and we use the short notation $E_{m, r}[\cdot]=E^{Q}\left[\cdot \mid R\left(\tau_{m}\right)=r\right]$. We also denote $v\left(\tau_{m}, r\right)$ by $v_{m}(r)$ and $\rho\left(r, \tau_{m}, t_{m}-\tau_{m}\right)$ by $\rho_{m}(r)$. The ex-coupon holding value at time $\tau_{m}$ when $R\left(\tau_{m}\right)=r$, denoted $v_{m}^{h}(r)$, can be written as

$$
v_{m}^{h}(r)=E_{m, r}\left[v_{m+1}\left(R\left(\tau_{m+1}\right)\right) \exp \left(-\int_{\tau_{m}}^{\tau_{m+1}} R(t) \mathrm{d} t\right)\right]
$$

for $m=n^{*}, \ldots n$, and

$$
v_{0}^{h}(r)=E_{0, r}\left[v_{n^{*}}\left(R\left(\tau_{n^{*}}\right)\right) \exp \left(-\int_{0}^{\tau_{n^{*}}} R(t) \mathrm{d} t\right)\right] .
$$

For $m=n^{*}, \ldots, n$, by the no-arbitrage principle of asset pricing, the expected benefit from exercising or holding the bond with its embedded options at time $\tau_{m}$ is given by

$$
\begin{array}{ll}
\left(C_{m}+c\right) \rho_{m}(r) & \text { if the issuer calls, } \\
v_{m}^{h}(r)+c \rho_{m}(r) & \text { if the investor holds, } \\
\left(P_{m}+c\right) \rho_{m}(r) & \text { if the investor puts. }
\end{array}
$$

The optimal exercising strategies are as follows. The issuer should call the bond at $\tau_{m}$ if its holding value exceeds the exercise benefit, i.e., if

$$
v_{m}^{h}(r)>C_{m} \rho_{m}(r) .
$$

On the other hand, the investor should put the bond at $\tau_{m}$ if the exercise benefit exceeds the holding value, i.e., if

$$
v_{m}^{h}(r)<P_{m} \rho_{m}(r) .
$$

Otherwise, the bond should be held for at least another period otherwise, i.e., if

$$
P_{m} \rho_{m}(r) \leqslant v_{m}^{h}(r) \leqslant C_{m} \rho_{m}(r) .
$$

Notice that it cannot be optimal for the issuer to call and for the investor to put simultaneously, since $0 \leqslant P_{m} \leqslant C_{m}$.

Assembling all these ingredients, we find that

$$
\begin{aligned}
& v_{n+1}(r)=1+c, \\
& v_{m}(r)=\max \left\{P_{m} \rho_{m}(r), \min \left(C_{m} \rho_{m}(r), v_{m}^{h}(r)\right)\right\}+c \rho_{m}(r) \\
& \text { for } m=n^{*}, \ldots, n \text {, } \\
& v_{0}(r)=v_{0}^{h}(r)+c \sum_{m=1}^{n^{*}} \rho_{m}(r) .
\end{aligned}
$$

Eqs. (2)-(6) are the DP recurrence equations. Solving these equations backwards from the maturity date to the origin (i.e., computing the functions $v_{m}^{h}$ and $v_{m}$ successively for $m=n, n-1, \ldots, 0)$ yields both the initial value of the bond and the optimal exercise strategies of its embedded options at all exercise dates, via the functions $v_{m}^{h}$. However, these functions do not have an analytical form, so they must 
be approximated numerically. An approximation method is detailed in the next section.

Büttler and Waldvogel (1996) also propose a DP approach, but they represent the value function $v_{m}^{h}$ as

$$
v_{m}^{h}(r)=\int_{-\infty}^{\infty} G\left(r, r^{\prime}, \tau_{m}, \tau_{m+1}\right) v_{m+1}\left(r^{\prime}\right) \mathrm{d} r^{\prime},
$$

where $G$ is a Green's function defined by

$$
G\left(r, r^{\prime}, \tau_{m}, \tau_{m+1}\right)=E_{m, r}\left[\delta\left(R\left(\tau_{m+1}\right)-r^{\prime}\right) \exp \left(-\int_{\tau_{m}}^{\tau_{m+1}} R(t) \mathrm{d} t\right)\right],
$$

in which $\delta$ is the Dirac delta function. The Green's function can be thought as representing the Arrow-Debreu state prices. This representation of $v_{m}^{h}$ is mathematically equivalent to ours. The difference between the two approaches resides in the approximation method: While BW compute $G$ in closed form and approximate $v_{m}^{h}$ by numerical integration, we approximate $v_{m}^{h}$ by a piecewise linear interpolation and integrate it in closed form. This is a key ingredient for the efficiency of our method.

\subsection{Approximation procedure}

The general idea is to start from the function $v_{n+1}$, which is a known constant, use it in (2) to compute $v_{n}^{h}(r)$ at a finite number of values of $r$, use (5) to get $v_{n}(r)$ at these values of $r$, and interpolate these values to obtain an approximation $\widehat{v}_{n}^{h}$ of $v_{n}^{h}$. This can be repeated iteratively for $m=n-1, \ldots, n^{*}$ : for a finite number of values of $r$, use the approximation $\widehat{v}_{m+1}$ of $v_{m+1}$ in (2) to compute an approximation of $v_{m}^{h}(r)$, put it in (5) to get an approximation $\widehat{v}_{m}(r)$ of $v_{m}(r)$, and interpolate these values to obtain an approximation $\widehat{v}_{m}$ of $v_{m}$. Do it again for $m=0$, but using (3) and (6) instead of (2) and (5). Note that the functions $v_{m}^{h}$ and $v_{m}$ are continuous for all $m$. In general, the expectation (or integral) in (2) can be approximated numerically for each $r$ of interest, but we shall introduce shortcuts that can dramatically reduce the computing time at that stage by avoiding the explicit numerical integration.

There are, of course, many ways of defining the interpolations $\widehat{v}_{m}$ for each $m$ (e.g., Hermite interpolation, splines, wavelets). Here, we use one of the simplest possible methods: piecewise-linear interpolation between the evaluation points. The main advantage of this simple method is that it allows us, under the dynamics presented here, to express the expectations in (2)-(3) in closed-form when $v_{m+1}$ is replaced by its piecewise-linear approximation.

To be more specific, let $r_{0}<r_{1}<\cdots<r_{p}<r_{p+1}=\infty$ be a fixed set of points forming a partition of $\mathbb{R}$. These are the values of $r$ at which the functions $v_{m}$ will be evaluated for the interpolation. The piecewise-linear approximation of $v_{m}$ has the form

$$
\widehat{v}_{m}(r)= \begin{cases}0 & \text { for } r<r_{0} \\ \alpha_{i}^{m}+\beta_{i}^{m} r & \text { for } r_{i} \leqslant r \leqslant r_{i+1}, \quad i=0, \ldots, p\end{cases}
$$


for some real-valued coefficients $\alpha_{i}^{m}$ and $\beta_{i}^{m}$, for $i=0, \ldots, p$, with $\beta_{p}^{m}=0$. After computing the value of $\widehat{v}_{m}$ at $r_{0}, \ldots, r_{p}$, the $2(p+1)$ coefficients $\alpha_{i}^{m}$ and $\beta_{i}^{m}$ are easily obtained by solving the following system of $2(p+1)$ linear equations:

$$
\begin{aligned}
& \widehat{v}_{m}\left(r_{0}\right)=\alpha_{0}^{m}+\beta_{0}^{m} r_{0}, \\
& \widehat{v}_{m}\left(r_{i}\right)=\alpha_{i}^{m}+\beta_{i}^{m} r_{i}=\alpha_{i-1}^{m}+\beta_{i-1}^{m} r_{i} \text { for } i=1, \ldots, p, \\
& 0=\beta_{p}^{m},
\end{aligned}
$$

whose solution can be written explicitly as

$$
\begin{aligned}
& \beta_{p}^{m}=0, \\
& \alpha_{p}^{m}=\widehat{v}_{m}\left(r_{p}\right), \\
& \beta_{i}^{m}=\left(\widehat{v}_{m}\left(r_{i+1}\right)-\widehat{v}_{m}\left(r_{i}\right)\right) /\left(r_{i+1}-r_{i}\right), \\
& \alpha_{i}^{m}=\left(r_{i+1} \widehat{v}_{m}\left(r_{i}\right)-r_{i} \widehat{v}_{m}\left(r_{i+1}\right)\right) /\left(r_{i+1}-r_{i}\right),
\end{aligned}
$$

for $i=1, \ldots, p-1$. We allow $a_{0}=-\infty$; in that case we have $\beta_{1}^{m}=0$ and $\alpha_{1}^{m}=\widehat{v}_{m}\left(r_{1}\right)$. The resulting function $\widehat{v}_{m}$ is continuous everywhere, except perhaps at $r_{0}$ if $\widehat{v}_{m}\left(r_{0}\right) \neq 0$.

An equivalent representation, in terms of a set of basis functions $N_{i}$, is

$$
\widehat{v}_{m}(r)=\sum_{i=0}^{p} N_{i}(r) \widehat{v}_{m}\left(r_{i}\right)
$$

where each $N_{i}$ is a hat function defined as the broken line that interpolates the three points $\left(r_{i-1}, 0\right),\left(r_{i}, 1\right),\left(r_{i+1}, 0\right)$, and is zero elsewhere, with the exception of $N_{0}$, which is linear between the points $\left(r_{0}, 1\right)$ and $\left(r_{1}, 0\right)$ and is zero elsewhere, and $N_{p}$, which is linear between the points $\left(r_{p-1}, 0\right)$ and $\left(r_{p}, 1\right)$, and equals 1 on the right of $\left(r_{p}, 1\right)$.

If we insert in (2) the piecewise-linear approximation (9) or (10) of $\widehat{v}_{m+1}$, we obtain the following approximation of $v_{m}^{h}\left(r_{k}\right)$ at any grid point $r_{k}$ :

$$
\begin{aligned}
\widehat{v}_{m}^{h}\left(r_{k}\right) & =E_{m, r_{k}}\left[\exp \left(-\int_{\tau_{m}}^{\tau_{m+1}} R(t) \mathrm{d} t\right) \widehat{v}_{m+1}\left(R\left(\tau_{m+1}\right)\right)\right] \\
& =\sum_{i=0}^{p} \widehat{v}_{m+1}\left(r_{i}\right) C_{k, i}^{m}
\end{aligned}
$$

for $m=n^{*}, \ldots n$ and similarly for $m=0$, where the constants $C_{k, i}^{m}$ can be precomputed once for all:

$$
C_{k, i}^{m}=\frac{B_{k, i-1}^{m}-r_{i-1} A_{k, i-1}^{m}}{r_{i}-r_{i-1}}+\frac{r_{i+1} A_{k, i}^{m}-B_{k, i}^{m}}{r_{i+1}-r_{i}}
$$


for all $m$, where

$$
\begin{aligned}
& A_{k, i}^{m}=E_{m, r_{k}}\left[\exp \left(-\int_{\tau_{m}}^{\tau_{m+1}} R(t) \mathrm{d} t\right) I\left(r_{i} \leqslant R\left(\tau_{m+1}\right)<r_{i+1}\right)\right], \\
& A_{k, i}^{0}=E_{0, r_{k}}\left[\exp \left(-\int_{\tau_{0}}^{\tau_{n^{*}}} R(t) \mathrm{d} t\right) I\left(r_{i} \leqslant R\left(\tau_{n^{*}}\right)<r_{i+1}\right)\right], \\
& B_{k, i}^{m}=E_{m, r_{k}}\left[\exp \left(-\int_{\tau_{m}}^{\tau_{m+1}} R(t) \mathrm{d} t\right) R\left(\tau_{m+1}\right) I\left(r_{i} \leqslant R\left(\tau_{m+1}\right)<r_{i+1}\right)\right], \\
& B_{k, i}^{0}=E_{0, r_{k}}\left[\exp \left(-\int_{\tau_{0}}^{\tau_{n^{*}}} R(t) \mathrm{d} t\right) R\left(\tau_{n^{*}}\right) I\left(r_{i} \leqslant R\left(\tau_{n^{*}}\right)<r_{i+1}\right)\right] .
\end{aligned}
$$

In the next section, we obtain closed-form formulas for the constants (or transition parameters) $A_{k, i}^{m}$ and $B_{k, i}^{m}$ for the Vasicek, generalized Vasicek, and CIR models. For the Vasicek and CIR models, these constants depend on $m$ only through $\tau_{m+1}-\tau_{m}$, and the expected discount factor $\rho_{m}\left(r_{k}\right)$ (for which a closed-form formula is also available) depends on $\tau_{m}$ and $t_{m}$ only through $t_{m}-\tau_{m}$, which are constant for $m \geqslant n^{*}$ in our models. The availability of these closed-form formulas and the fact that the constants do not depend on $m$ is a key ingredient for the efficiency of our DP procedure. The piecewise-linear approximation of the function $v_{m}$ was chosen precisely for that reason.

\section{Conditional distributions and expectations for specific models}

To derive formulas for the constants $A_{k, i}^{m}$ and $B_{k, i}^{m}$ defined in (11), for the Vasicek, generalized Vasicek, and CIR models, we start from the distribution of the random vector

$$
\left(R(\tau+\delta), \int_{\tau}^{\tau+\delta} R(t) \mathrm{d} t\right)
$$

conditional on the value of $R(\tau)$, for $0 \leqslant \tau \leqslant \tau+\delta \leqslant T$. The formulas for the coefficients $A_{k, i}^{m}, B_{k, i}^{m}$ and $\rho\left(r_{k}, \tau_{m}, \delta\right)$ follow as corollaries.

\subsection{The Vasicek model}

Under the risk-neutral probability measure, the interest-rate process is the solution to the following stochastic differential equation

$$
\mathrm{d} R(t)=\kappa(\bar{r}-R(t)) \mathrm{d} t+\sigma \mathrm{d} B(t) \text { for } 0 \leqslant t \leqslant T .
$$

Closed-form solutions for prices of zero-coupon bonds and European bond options are available in this model (see, e.g., Jamshidian, 1989).

Theorem 1. For the Vasicek model, the distribution of the random vector (11) conditional on $R(\tau)=r$ is bivariate normal with mean

$$
\mu(r, \delta)=\left(\mu_{1}(r, \delta), \mu_{2}(r, \delta)\right)=\left(\bar{r}+\mathrm{e}^{-\kappa \delta}(r-\bar{r}), \bar{r} \delta+\frac{1-\mathrm{e}^{-\kappa \delta}}{\kappa}(r-\bar{r})\right)
$$


and covariance matrix

$$
\Sigma(\delta)=\left[\begin{array}{ll}
\sigma_{1}^{2}(\delta) & \sigma_{12}(\delta) \\
\sigma_{21}(\delta) & \sigma_{2}^{2}(\delta)
\end{array}\right]=\left[\begin{array}{ll}
\frac{\sigma^{2}}{2 \kappa}\left(1-\mathrm{e}^{-2 \kappa \delta}\right) & \frac{\sigma^{2}}{2 \kappa^{2}}\left(1-2 \mathrm{e}^{-\kappa \delta}+\mathrm{e}^{-2 \kappa \delta}\right) \\
\sigma_{12}(\delta) & \frac{\sigma^{2}}{2 \kappa^{3}}\left(-3+2 \kappa \delta+4 \mathrm{e}^{-\kappa \delta}-\mathrm{e}^{-2 \kappa \delta}\right)
\end{array}\right]
$$

Proof. By applying Itô's lemma to the process $\left\{\xi(t, R(t))=\mathrm{e}^{\kappa t} R(t), 0 \leqslant t \leqslant T\right\}$, we obtain that for $0 \leqslant \tau \leqslant t \leqslant u$,

$$
R(u)=\bar{r}+\mathrm{e}^{-\kappa(u-\tau)}(r-\bar{r})+\sigma \int_{\tau}^{u} \mathrm{e}^{-\kappa(u-t)} \mathrm{d} B(t),
$$

and consequently that

$$
\begin{aligned}
\int_{\tau}^{\tau+\delta} R(u) \mathrm{d} u & =\bar{r} \delta+\frac{1-\mathrm{e}^{-\kappa \delta}}{\kappa}(r-\bar{r})+\sigma \int_{\tau}^{\tau+\delta}\left(\int_{\tau}^{u} \mathrm{e}^{-\kappa(u-t)} \mathrm{d} B(t)\right) \mathrm{d} u \\
& =\bar{r} \delta+\frac{1-\mathrm{e}^{-\kappa \delta}}{\kappa}(r-\bar{r})+\sigma \int_{\tau}^{\tau+\delta}\left(\int_{t}^{\tau+\delta} \mathrm{e}^{-\kappa(u-t)} \mathrm{d} u\right) \mathrm{d} B(t),
\end{aligned}
$$

where the last equality comes from Lemma 6 of the appendix. Conditioning on the information available at time $t$, we can decompose $R(\tau+\delta)$ and $\int_{\tau}^{\tau+\delta} R(t) \mathrm{d} t$ into a deterministic part and a random part. The random part turns out to be a limit of linear combinations of the same standard Brownian motion taken at different points in time. The random variables $R(\tau+\delta)$ and $\int_{\tau}^{\tau+\delta} R(t) \mathrm{d} t$, conditional on $R(\tau)$, are thus jointly normal.

The mean vector and covariance matrix of the normally distributed random vector

$$
\left(R(\tau+\delta), \int_{\tau}^{\tau+\delta} R(t) \mathrm{d} t\right)
$$

conditional on $R(\tau)$, can then be derived from the basic properties of stochastic integrals (e.g., Øksendal, 1995). This is done in Lemma 7 of the appendix.

Corollary 2. For the Vasicek model, the transition parameters and discount factor are given by

$$
\begin{aligned}
& \rho\left(r_{k}, \tau_{m}, \delta\right)=\exp \left(-\mu_{2}(r, \delta)+\sigma_{2}^{2}(\delta) / 2\right), \\
& A_{k, i}^{m}=\mathrm{e}^{-\mu_{2}\left(r_{k}, \delta\right)+\sigma_{2}^{2}(\delta) / 2}\left[\Phi\left(x_{k, i}\right)-\Phi\left(x_{k, i-1}\right)\right]
\end{aligned}
$$

and

$$
\begin{aligned}
B_{k, i}^{m}= & \mathrm{e}^{-\mu_{2}\left(r_{k}, \delta\right)+\sigma_{2}^{2}(\delta) / 2}\left[\left(\mu_{1}\left(r_{k}, \delta\right)-\sigma_{12}(\delta)\right)\left(\Phi\left(x_{k, i}\right)-\Phi\left(x_{k, i-1}\right)\right)\right. \\
& \left.-\sigma_{1}(\delta)\left(\mathrm{e}^{-x_{k, i}^{2}}-\mathrm{e}^{-x_{k, i-1}^{2}}\right) / \sqrt{2 \pi}\right]
\end{aligned}
$$

where

$$
\begin{aligned}
& x_{k, i}=\left(r_{i}-\mu_{1}\left(r_{k}, \delta\right)+\sigma_{12(\delta)}\right) / \sigma_{1} \text { for } i=0, \ldots, p, \\
& x_{k,-1}=-\infty
\end{aligned}
$$


the $\mu_{i}\left(r_{k}, \delta\right)$ and the $\sigma_{i j}(\delta)$ are given in (13) and (14) with $\delta=\tau_{m+1}-\tau_{m}$ for $m=$ $n^{*}, \ldots, n$ and $\delta=\tau_{n^{*}}-\tau_{0}$ for $m=0$, and $\Phi$ is the standard normal distribution function.

Proof. Using Theorem 1, the derivation is straightforward.

\subsection{The generalized Vasicek model}

We now consider a special case of the generalized Vasicek model with time dependent long run mean, proposed by Hull and White (1990a). Under the riskneutral probability measure, the short rate process is the solution to the stochastic differential equation

$$
\mathrm{d} R(t)=\kappa(\bar{r}(t)-R(t)) \mathrm{d} t+\sigma \mathrm{d} B(t) \quad \text { for } 0 \leqslant t \leqslant T .
$$

This model requires the use of market data by fitting the zero-coupon yield curve. This can easily be done using, for instance, the Nelson and Siegel (1987) model. Once this is done, it provides an exact fit to the initial term structure and has a closed-form solution for the long run mean $\bar{r}(t)$, given by

$$
\bar{r}(t)=-\frac{1}{\kappa} \frac{\partial^{2} \log (P(t))}{\partial t^{2}}-\frac{\partial \log (P(t))}{\partial t}+\frac{\sigma^{2}}{2 \kappa^{2}}\left(1-\mathrm{e}^{-2 \kappa t}\right),
$$

where $P(t)$ denotes the function fitted to the observed prices at time 0 of zero-coupon bonds with maturity $t$.

Closed form solutions for prices of zero-coupon bonds and European bond options are also available in this model (see, e.g., Hull and White, 1990a).

Theorem 3. For the generalized Vasicek model, the distribution of the random vector (11) conditional on $R(\tau)=r$ is bivariate normal with mean

$$
\mu^{\star}(r, \delta)=\left(\mu_{1}^{\star}(r, \delta), \mu_{2}^{\star}(r, \delta)\right),
$$

where

$$
\begin{aligned}
\mu_{1}^{\star}(r, \delta)= & r \mathrm{e}^{-\kappa \delta}-\left.\frac{\partial \log (P(t))}{\partial t}\right|_{\tau+\delta}+\left.\mathrm{e}^{-\kappa \delta} \frac{\partial \log (P(t))}{\partial t}\right|_{\tau} \\
& +\frac{\sigma^{2}}{2 \kappa^{2}}\left(1-\mathrm{e}^{-\kappa \delta}\right)+\frac{\sigma^{2}}{2 \kappa^{2}} \mathrm{e}^{-\kappa \tau}\left(\mathrm{e}^{-\kappa(\tau+\delta)}-\mathrm{e}^{-\kappa \tau}\right), \\
\mu_{2}^{\star}(r, \delta)= & r \frac{\left(1-\mathrm{e}^{-\kappa \delta}\right)}{\kappa}-\log \left(\frac{P(\tau+\delta)}{P(\tau)}\right)+\left.\frac{\partial \log (P(t))}{\partial t}\right|_{\tau} \frac{\left(1-\mathrm{e}^{-\kappa \delta}\right)}{\kappa} \\
& +\frac{\sigma^{2}}{2 \kappa^{2}} \delta-\frac{\sigma^{2}}{2 \kappa^{3}}\left(1-\mathrm{e}^{-\kappa \delta}\right)+\frac{\sigma^{2}}{4 \kappa^{3}}\left(\mathrm{e}^{-\kappa(\tau+\delta)}-\mathrm{e}^{-\kappa \tau}\right)^{2},
\end{aligned}
$$

and the same covariance matrix $\Sigma(\delta)$ as in Theorem 1 . 
Proof. The joint normality of $\left(R(\tau+\delta), \int_{\tau}^{\tau+\delta} R(t) \mathrm{d} t\right)$ conditional on $R(\tau)$ can be proved by a similar argument as in Theorem 1 . Stochastic integration then yields the mean and covariance matrix of this conditional distribution.

More specifically, by applying Itô's lemma to the process $\{\xi(t, R(t))=$ $\left.\mathrm{e}^{\kappa t} R(t), 0 \leqslant t \leqslant T\right\}$, we obtain that

$$
R(u)=R(\tau) \mathrm{e}^{-\kappa(u-\tau)}+\int_{\tau}^{u} \kappa \bar{r}(t) \mathrm{e}^{-\kappa(u-s)} \mathrm{d} t+\sigma \int_{\tau}^{u} \mathrm{e}^{-\kappa(u-t)} \mathrm{d} B(t) .
$$

This implies that

$$
\begin{aligned}
\mu_{1}^{\star}(r, \delta)= & r \mathrm{e}^{-\kappa \delta}+\int_{\tau}^{\tau+\delta} \kappa \bar{r}(t) \mathrm{e}^{-\kappa(\tau+\delta-t)} \mathrm{d} t \\
= & r \mathrm{e}^{-\kappa \delta}+\int_{\tau}^{\tau+\delta}\left(\kappa-\frac{1}{\kappa} \frac{\partial^{2} \log (P(t))}{\partial t^{2}}-\frac{\partial \log (P(t))}{\partial t}\right. \\
& \left.+\frac{\sigma^{2}}{2 \kappa^{2}}\left(1-\mathrm{e}^{-2 \kappa t}\right) \mathrm{e}^{-\kappa(\tau+\delta-t)}\right) \mathrm{d} t \\
= & r \mathrm{e}^{-\kappa \delta}-\left.\frac{\partial \log (P(t))}{\partial t}\right|_{\tau+\delta}+\left.\mathrm{e}^{-\kappa \delta} \frac{\partial \log (P(t))}{\partial t}\right|_{\tau} \\
& +\frac{\sigma^{2}}{2 \kappa^{2}}\left(1-\mathrm{e}^{-\kappa \delta}\right)+\frac{\sigma^{2}}{2 \kappa^{2}} \mathrm{e}^{-\kappa(\tau+\delta)}\left(\mathrm{e}^{-\kappa(\tau+\delta)}-\mathrm{e}^{-\kappa \tau}\right) .
\end{aligned}
$$

The conditional mean $\mu_{2}^{\star}(r, \delta)$ can then be computed as follows:

$$
\begin{aligned}
\mu_{2}^{\star}(r, \delta)= & E\left[\int_{\tau}^{\tau+\delta} R(t) \mathrm{d} t \mid R(\tau)=r\right] \\
= & \int_{\tau}^{\tau+\delta} E[R(t) \mid R(\tau)=r] \mathrm{d} t \\
= & \int_{\tau}^{\tau+\delta}\left(r \mathrm{e}^{-\kappa(t-\tau)}-\frac{\partial \log (P(t))}{\partial t}+\left.\mathrm{e}^{-\kappa(t-\tau)} \frac{\partial \log (P(t))}{\partial t}\right|_{\tau}\right. \\
& \left.+\frac{\sigma^{2}}{2 \kappa^{2}}\left(1-\mathrm{e}^{-\kappa(t-\tau)}\right)+\frac{\sigma^{2}}{2 \kappa^{2}} \mathrm{e}^{-\kappa t}\left(\mathrm{e}^{-\kappa t}-\mathrm{e}^{-\kappa \tau}\right)\right) \mathrm{d} t \\
= & r \frac{\left(1-\mathrm{e}^{-\kappa \delta}\right)}{\kappa}-\log \left(\frac{P(\tau+\delta)}{P(\tau)}\right)+\left.\frac{\partial \log (P(t))}{\partial t}\right|_{\tau} \frac{\left(1-\mathrm{e}^{-\kappa \delta}\right)}{\kappa} \\
& +\frac{\sigma^{2}}{2 \kappa^{2}} \delta-\frac{\sigma^{2}}{2 \kappa^{3}}\left(1-\mathrm{e}^{-\kappa \delta}\right)+\frac{\sigma^{2}}{4 \kappa^{3}}\left(\mathrm{e}^{-\kappa(\tau+\delta)}-\mathrm{e}^{-\kappa \tau}\right)^{2} .
\end{aligned}
$$

The conditional covariance matrix is the same as in Theorem 1 and can be derived in the same way.

Corollary 4. For the generalized Vasicek model, the discount factor and the transition parameters are given by the same expressions as in Corollary 2 , but with $\mu$ replaced by $\mu^{\star}$. 
Proof. The derivation is straightforward from Theorem 3 and we omit the details.

\subsection{The CIR model}

Under the risk-neutral probability measure, the short rate process is the solution to the following stochastic differential equation:

$$
\mathrm{d} R(t)=\kappa(\bar{r}-R(t)) \mathrm{d} t+\sigma \sqrt{R(t)} \mathrm{d} B(t) \quad \text { for } 0 \leqslant t \leqslant T .
$$

In this model, the joint distribution of the random vector in (11) conditional on $R(\tau)=r$ is characterized by its Laplace transform (see, e.g., Feller, 1951, or Lamberton and Lapeyre, 1996, p. 126):

$$
\begin{aligned}
& E\left[\exp \left(-\omega \int_{\tau}^{\tau+\delta} R(t) \mathrm{d} t-v R(\tau+\delta)\right) \mid R(\tau)=r\right] \\
& \quad=\exp (X(r, \delta, \omega, v)-Y(r, \delta, \omega, v) r),
\end{aligned}
$$

where

$$
\begin{aligned}
& \gamma(\omega)=\sqrt{\kappa^{2}+2 \omega \sigma^{2}}, \\
& X(\delta, \omega, v)=\frac{2 \kappa \bar{r}}{\sigma^{2}} \log \left[\frac{2 \gamma(\omega) \mathrm{e}^{(\gamma(\omega)+\kappa) \delta / 2}}{\left(v \sigma^{2}+\gamma(\omega)+\kappa\right)\left(\mathrm{e}^{\gamma(\omega) \delta}-1\right)+2 \gamma(\omega)}\right], \\
& Y(\delta, \omega, v)=\frac{v\left(\gamma(\omega)+\kappa+\mathrm{e}^{\gamma(\omega) \delta}(\gamma(\omega)-\kappa)\right)+2 \omega\left(\mathrm{e}^{\gamma(\omega) \delta}-1\right)}{\left(v \sigma^{2}+\gamma(\omega)+\kappa\right)\left(\mathrm{e}^{\gamma(\omega) \delta}-1\right)+2 \gamma(\omega)} .
\end{aligned}
$$

When $\omega=1$ and $v=0$, the Laplace transform gives the price of a zero-coupon bond. We also use this characterization to obtain the following proposition, whose proof is given in the appendix.

Proposition 5. For the CIR model, the discount factor and transition parameters are given by

$$
\begin{aligned}
& \rho\left(r_{k}, \tau_{m}, \delta\right)=\exp \left(X\left(r_{k}, \delta, 1,0\right)-Y\left(r_{k}, \delta, 1,0\right) r\right), \\
& A_{k, i}^{m}=\rho\left(r_{k}, \tau_{m}, \delta\right) \sum_{t=0}^{\infty} \mathrm{e}^{-\lambda_{k} / 2} \frac{\left(\lambda_{k} / 2\right)^{n}}{t !}\left(F_{d+2 t}\left(\frac{r_{i+1}}{\eta}\right)-F_{d+2 t}\left(\frac{r_{i}}{\eta}\right)\right),
\end{aligned}
$$

and

$$
\begin{aligned}
B_{k, i}^{m}= & \rho\left(r_{k}, \tau_{m}, \delta\right) \eta \sum_{t=0}^{\infty} \mathrm{e}^{-\lambda_{k} / 2} \frac{\left(\lambda_{k} / 2\right)^{t}}{t !}\left[-2\left(r_{i+1} f_{d+2 t}\left(r_{i+1} / \eta\right)-r_{i} f_{d+2 t}\left(r_{i} / \eta\right)\right)\right. \\
& \left.+(d+2 t)\left(F_{d+2 t}\left(r_{i+1} / \eta\right)-F_{d+2 t}\left(r_{i} / \eta\right)\right)\right],
\end{aligned}
$$

for $i=0, \ldots, p$, where $F_{d+2 t}$ and $f_{d+2 t}$ are the distribution and density functions of a chi-square random variable with $d+2 t$ degrees of freedom, $X(\cdot), Y(\cdot)$ are 
defined in (19),

$$
\begin{aligned}
\delta & =\tau_{m+1}-\tau_{m} \quad \text { for } m=n^{*}, \ldots n, \\
\delta & =\tau_{n^{*}}-\tau_{0} \text { at } m=0, \\
\gamma & =\sqrt{\kappa^{2}+2 \sigma^{2}} \\
\eta & =\frac{\sigma^{2}\left(\mathrm{e}^{\gamma \delta}-1\right)}{2(\gamma+\kappa)\left(\mathrm{e}^{\gamma \delta}-1\right)+2 \gamma}, \\
d & =\frac{4 \kappa \bar{r}}{\sigma^{2}}
\end{aligned}
$$

and

$$
\lambda_{k}=\frac{8 \gamma^{2} \mathrm{e}^{\gamma \delta} r_{k}}{\sigma^{2}\left[(\gamma+\kappa)\left(\mathrm{e}^{\gamma \delta}-1\right)+2 \gamma\right]\left(\mathrm{e}^{\gamma \delta}-1\right)} .
$$

\section{Numerical illustrations}

In this section, we compare the performance of our DP procedure with that of the methods proposed by BW and DFVL on a numerical example taken from BW and also considered by DFVL. The comparisons are made with both the Vasicek and CIR interest-rate models.

The security to be priced is a $4.25 \%$ callable bond issued by the Swiss Confederation, over the period 1987-2012. At the pricing date $t_{0}=0$, December 23, 1991, the time to maturity was $T=t_{n}=20.172$ years, with $n=21$, a principal scaled to 1 , a coupon $c=0.0425$ coming once a year with the first coupon coming at time $t_{1}=0.172$, a notice period of 2 months, i.e., $\Delta t=t_{m}-\tau_{m}=0.1666$, and a protection period of $t_{n^{*}}=10.172$ years, with $n^{*}=11$. The call prices are $C_{11}=1.025, \quad C_{12}=1.020, \quad C_{13}=1.015, \quad C_{14}=1.010, \quad C_{15}=1.005$, and $C_{16}=$ $\cdots=C_{21}=1$.

The values of the parameters $\bar{r}, \kappa, \sigma$, given in Table 1 , are taken from BW.

The grid points $r_{1}, \ldots, r_{p}$ are selected to be equally spaced with $r_{0}=-\infty, r_{1}=$ $\bar{r}-6 \sigma_{1}$ and $r_{p}=\bar{r}+6 \sigma_{1}$ for the Vasicek model, whereas $r_{0}=0, r_{1}=10^{-6}$ and $r_{p}=3$, for the CIR model.

We first compare the accuracies of the three methods on a simple case where a closed-form solution is available for the exact value: the straight bond, without any

Table 1

Input data for the Vasicek and CIR models

\begin{tabular}{lll}
\hline & Vasicek & CIR \\
\hline $\bar{r}$ & 0.098397028 & 0.133976855 \\
$\kappa$ & 0.44178462 & 0.14294371 \\
$\sigma$ & 0.13264223 & 0.38757496 \\
\hline
\end{tabular}


Table 2

Convergence of our DP procedure: value of $v_{0}(r)$ computed by several methods, for the Vasicek model

Values of the straight bonds for Vasicek model

\begin{tabular}{lllllll}
\hline$r$ & $p=600$ & $p=1200$ & $p=2400$ & Formula & BW & DFVL \\
\hline 0.01 & 0.92746 & 0.92743 & 0.92742 & 0.92742 & 0.9274 & 0.92739 \\
0.02 & 0.90899 & 0.90896 & 0.90896 & 0.90895 & 0.9089 & 0.90892 \\
0.03 & 0.89091 & 0.89089 & 0.89088 & 0.89088 & 0.8908 & 0.89084 \\
0.04 & 0.87322 & 0.87319 & 0.87319 & 0.87318 & 0.8731 & 0.87315 \\
0.05 & 0.85590 & 0.85588 & 0.85587 & 0.85587 & 0.8558 & 0.85583 \\
0.06 & 0.83895 & 0.83893 & 0.83892 & 0.83892 & 0.8389 & 0.83887 \\
0.07 & 0.82236 & 0.82233 & 0.82233 & 0.82233 & 0.8223 & 0.82228 \\
0.08 & 0.80612 & 0.80610 & 0.80609 & 0.80609 & 0.8060 & 0.80604 \\
0.09 & 0.79022 & 0.79020 & 0.79020 & 0.79019 & 0.7901 & 0.79014 \\
0.10 & 0.77466 & 0.77464 & 0.77464 & 0.77464 & 0.7746 & 0.77458 \\
\hline
\end{tabular}

embedded options. In other words, we assume that $n^{*}=n$ and keep the values given above for all other parameters. Tables 1 and 2 report the results for the Vasicek and CIR models, respectively; they give the estimated values of $v_{0}(r)$ with different methods. The column "formula" gives the exact value obtained from the closed-form formula. The last two columns report the values obtained by BW and DFVL in their papers. Columns 2-4 give the approximations obtained by our DP procedure (denoted by BBKL), for grid sizes with $p=600,1200$, and 2400 . We find that our DP approximations converge very nicely to the exact values as $p$ increases. The precision of our method compares advantageously with DFVL and BW. BW reported their results with only four digits of accuracy.

Since we are approximating a continuous function by a piecewise linear interpolant, the approximation error at each step should converge as $\mathrm{O}\left(\Delta^{2}\right)$, where $\Delta=\max _{i}\left(r_{i+1}-r_{i}\right)$. The total error on $v_{0}(r)$ is the cumulation of this approximation error over the successive steps, combined with other sources of errors (e.g., in the numerical computation of the $A_{k, i}^{m}$ and $B_{k, i}^{m}$ ) which are deemed less important. Thus, we expect the pricing errors in Tables 1 and 2 to converge as $\mathrm{O}\left(\Delta^{2}\right)=\mathrm{O}\left(p^{-2}\right)$. We computed the exact error (by comparing with the formula) for $p=75,150, \ldots, 2400$, for each value of $r$, then plotted the log of this error as a function of $\log (\Delta)$. In all cases the numbers fitted roughly a straight line, with slope ranging from 1.93 to 2.15 . So doubling $p$ divides the error by 4 , approximately.

Table 3 reports the value of the callable bond, with its embedded options and $n^{*}=11$, obtained by the three approximation methods. Our DP results are for a grid size with $p=1200$. The results for $p=2400$ are practically the same as those reported in the table (with absolute error less than 0.00003 , comparable to the differences observed in Tables 1 and 2). This indicates that convergence has occurred. The results of DFVL and our method are close to each other, but the results of BW differ significantly for the Vasicek model. 
Table 3

Convergence of our DP procedure: value of $v_{0}(r)$ computed by several methods, for the CIR model

Values of the straight bonds for CIR model

\begin{tabular}{lllllll}
\hline$r$ & $p=600$ & $p=1200$ & $p=2400$ & Formula & BW & DFVL \\
\hline 0.01 & 0.95537 & 0.95528 & 0.95526 & 0.95525 & 0.9552 & 0.95527 \\
0.02 & 0.93166 & 0.93157 & 0.93154 & 0.93154 & 0.9315 & 0.93155 \\
0.03 & 0.90858 & 0.90848 & 0.90846 & 0.90845 & 0.9084 & 0.90846 \\
0.04 & 0.88610 & 0.88601 & 0.88599 & 0.88598 & 0.8859 & 0.88599 \\
0.05 & 0.86422 & 0.86414 & 0.86411 & 0.86411 & 0.8641 & 0.86411 \\
0.06 & 0.84292 & 0.84284 & 0.84282 & 0.84281 & 0.8428 & 0.84281 \\
0.07 & 0.82219 & 0.82211 & 0.82208 & 0.82208 & 0.8220 & 0.82207 \\
0.08 & 0.80200 & 0.80192 & 0.80190 & 0.80189 & 0.8018 & 0.80188 \\
0.09 & 0.78235 & 0.78227 & 0.78225 & 0.78224 & 0.7822 & 0.78223 \\
0.10 & 0.76322 & 0.76314 & 0.76312 & 0.76311 & 0.7631 & 0.76309 \\
\hline
\end{tabular}

Table 4

Bond values obtained by the three methods for the Vasicek and CIR models

Values of callable and putable bonds

\begin{tabular}{|c|c|c|c|c|c|c|}
\hline \multirow[b]{2}{*}{$r$} & \multicolumn{3}{|c|}{ Vasicek model } & \multicolumn{3}{|c|}{ CIR model } \\
\hline & BW & DFVL & BBKL & BW & DFVL & BBKL \\
\hline 0.01 & 0.8556 & 0.84282 & 0.84285 & 0.9392 & 0.93926 & 0.93921 \\
\hline 0.02 & 0.8338 & 0.82627 & 0.82630 & 0.9159 & 0.91598 & 0.91595 \\
\hline 0.03 & 0.8223 & 0.81010 & 0.81009 & 0.8933 & 0.89333 & 0.89330 \\
\hline 0.04 & 0.8062 & 0.79420 & 0.79423 & 0.8712 & 0.87127 & 0.87125 \\
\hline 0.05 & 0.7904 & 0.77868 & 0.77871 & 0.8498 & 0.84980 & 0.84978 \\
\hline 0.06 & 0.7749 & 0.76348 & 0.76351 & 0.8289 & 0.82890 & 0.82888 \\
\hline 0.07 & 0.7598 & 0.74860 & 0.74862 & 0.8085 & 0.80855 & 0.80854 \\
\hline 0.08 & 0.7450 & 0.73403 & 0.73406 & 0.7887 & 0.78874 & 0.78873 \\
\hline 0.09 & 0.7305 & 0.71977 & 0.71980 & 0.7694 & 0.76945 & 0.76945 \\
\hline 0.10 & 0.7163 & 0.70578 & 0.70583 & 0.7507 & 0.75067 & 0.75067 \\
\hline
\end{tabular}

Our code is written in C, compiled with gcc, and executed on a $2.0 \mathrm{GHz}$ Pentium 4 processor running under Windows XP. Our DP procedure takes between 2 and $3 \mathrm{~s}$ of CPU time, including all precomputations, to compute one entry of Table 3 for a grid size of $p=1200$. As an indication, CPU times reported by DFVL are between 12 and $14 \mathrm{~s}$ (on a Sun Ultra Sparc) and those reported by BW are over $200 \mathrm{~s}$.

The value of the corresponding embedded call option can be obtained by

$$
v_{0}^{\text {call option }}(r)=v_{0}^{\text {straight bond }}(r)-v_{0}^{\text {callable bond }}(r),
$$

where $r=R\left(t_{0}\right)$ is the current interest rate at $t_{0}$ (Table 4). 


\section{Conclusion}

In this paper, we model the valuation of options embedded in bonds as a stochastic dynamic programming problem. We propose a simple and efficient approximation for the case of three affine models of the term structure. Our method is based on a piecewise linear approximation of the value function and is quite easy to implement. It differs from the usual lattice-based and finite-difference methods in that the exact conditional probability distribution of the interest rate is used in order to compute the transition probabilities. We provide numerical results for the Vasicek and CIR models. These results demonstrate convergence and an efficiency that compares favorably with that of the other available methods. In addition, our methodology readily extends to handle the generalized Vasicek model with time-dependent longrun mean, provided that a function can be fitted to the observed zero-coupon bond prices. Extensive empirical studies of the term structure generated by callable and puttable bonds have been lacking in the literature, mainly due to the lack of a robust and efficient numerical procedure for pricing these bonds. Our approach can provide this type of numerical procedure for many situations, where it can be used to investigate empirically the cross-sectional and time series properties of bonds with embedded options. Our solution technique is flexible and can be extended to other models involving different continuous-time as well as discrete-time processes, whenever the transition parameters can be obtained efficiently. In particular, it can be adapted to models with multifactor affine term structure (Dai and Singleton, 2000) and more generally to the valuation of other Bermudan or American-style derivatives in the setting of affine diffusion state processes (Duffie et al., 2000, 2003).

\section{Acknowledgments}

This work has been supported by Natural Sciences and Engineering Research Council of Canada (NSERC) grants and an IFM $^{2}$ grant to the first two authors, as well as a NSERC grant, a NATEQ-Québec grant, and a Canada Research Chair to the fourth author. We thank the two anonymous reviewers, whose suggestions helped improving the presentation.

\section{Appendix A}

Lemma 6. If $f$ and $g$ are two real-valued functions continuously differentiable in $[\tau, \tau+\delta]$, for $0 \leqslant \tau \leqslant \tau+\delta \leqslant T$, and $\{B(t), t \geqslant 0\}$ is a standard Brownian motion, then

$$
\begin{aligned}
\int_{\tau}^{\tau+\delta} & \left(\int_{\tau}^{u} f(t) g(u) \mathrm{d} B(t)\right) \mathrm{d} u \\
\quad= & \int_{\tau}^{\tau+\delta}\left(\int_{\tau}^{\tau+\delta} f(t) g(u) I(t \in[\tau, u]) \mathrm{d} B(t)\right) \mathrm{d} u
\end{aligned}
$$




$$
\begin{aligned}
& =\int_{\tau}^{\tau+\delta}\left(\int_{\tau}^{\tau+\delta} f(t) g(u) I(u \in[t, \tau+\delta]) \mathrm{d} u\right) \mathrm{d} B(t) \\
& =\int_{\tau}^{\tau+\delta}\left(\int_{t}^{\tau+\delta} f(t) g(u) \mathrm{d} u\right) \mathrm{d} B(t) .
\end{aligned}
$$

Proof. This is a special case of Fubini's theorem; see, e.g., Protter (2004, p. 207).

Lemma 7. In Theorem 1, the mean vector and covariance matrix of the random vector $\left(R(\tau+\delta), \int_{\tau}^{\tau+\delta} R(t) \mathrm{d} t\right)$, conditional on $R(\tau)=r$, are given by (13) and (14).

Proof. The conditional mean is

$$
E\left[\left(R(\tau+\delta), \int_{\tau}^{\tau+\delta} R(t) \mathrm{d} t\right) \mid R(\tau)=r\right]=\left(\bar{r}+\mathrm{e}^{-\kappa \delta}(r-\bar{r}), \bar{r} \delta+\frac{1-\mathrm{e}^{-\kappa \delta}}{\kappa}(r-\bar{r})\right),
$$

since the centered random vector

$$
\left(\int_{\tau}^{\tau+\delta} \mathrm{e}^{-\kappa(u-t)} \mathrm{d} B(t), \int_{\tau}^{\tau+\delta}\left(\int_{t}^{\tau+\delta} \mathrm{e}^{-\kappa(u-t)} \mathrm{d} u\right) \mathrm{d} B(t)\right)
$$

is independent of $\{R(t), t \leqslant \tau\}$.

The conditional variance of $R(\tau+\delta)$ is

$$
\begin{aligned}
\operatorname{Var}[R(\tau+\delta) \mid R(\tau)=r] & =E\left[\left(\int_{\tau}^{\tau+\delta} \sigma \mathrm{e}^{-\kappa(\tau+\delta-t)} \mathrm{d} B(t)\right)^{2} \mid R(\tau)=r\right] \\
& =\sigma^{2} \int_{\tau}^{\tau+\delta} \mathrm{e}^{-2 \kappa(\tau+\delta-t)} \mathrm{d} t=\frac{\sigma^{2}}{2 \kappa}\left(1-\mathrm{e}^{-2 \kappa \delta}\right) .
\end{aligned}
$$

The conditional variance of $\int_{\tau}^{\tau+\delta} R(t) \mathrm{d} t$ is

$$
\begin{aligned}
\operatorname{Var} & {\left[\int_{\tau}^{\tau+\delta} R(t) \mathrm{d} t \mid R(\tau)=r\right] } \\
& =E\left[\left(\int_{\tau}^{\tau+\delta}\left(\int_{t}^{\tau+\delta} \sigma \mathrm{e}^{-\kappa(u-t)} \mathrm{d} u\right) \mathrm{d} B(t)\right)^{2} \mid R(\tau)=r\right] \\
& =\sigma^{2} \int_{\tau}^{\tau+\delta}\left(\int_{\tau}^{\tau+\delta} \mathrm{e}^{-\kappa(u-t)} \mathrm{d} u\right)^{2} \mathrm{~d} t=\frac{\sigma^{2}}{2 \kappa^{3}}\left(-3+2 \kappa \delta+4 \mathrm{e}^{-\kappa \delta}-\mathrm{e}^{-2 \kappa \delta}\right) .
\end{aligned}
$$

The conditional covariance between $R(\tau+\delta)$ and $\int_{\tau}^{\tau+\delta} R(t) \mathrm{d} t$ is

$$
\begin{aligned}
\operatorname{Cov} & {\left[R(\tau+\delta), \int_{\tau}^{\tau+\delta} R(t) \mathrm{d} t \mid R(\tau)=r\right] } \\
& =E\left[\int_{\tau}^{\tau+\delta} \sigma \mathrm{e}^{-\kappa(\tau+\delta-t)} \mathrm{d} B(t) \int_{\tau}^{\tau+\delta}\left(\int_{\tau}^{\tau+\delta} \sigma \mathrm{e}^{-\kappa(u-t)} \mathrm{d} u\right) \mathrm{d} B(t) \mid R(\tau)=r\right]
\end{aligned}
$$




$$
\begin{aligned}
& =\sigma^{2} \int_{\tau}^{\tau+\delta} \mathrm{e}^{-\kappa(\tau+\delta-t)}\left(\int_{t}^{\tau+\delta} \mathrm{e}^{-\kappa(u-t)} \mathrm{d} u\right) \mathrm{d} t \\
& =\frac{\sigma^{2}}{2 \kappa^{2}}\left(1-2 \mathrm{e}^{-\kappa \delta}+\mathrm{e}^{-2 \kappa \delta}\right) .
\end{aligned}
$$

Proof of Proposition 5. We start by defining a new probability measure $\widetilde{Q}$, known as the forward-neutral measure (see, e.g., Geman et al., 1995), such that:

$$
\frac{\mathrm{d} \widetilde{Q}}{\mathrm{~d} Q}=\frac{\mathrm{e}^{-\int_{\tau}^{\tau+\delta} R(t) \mathrm{d} t}}{E\left[\mathrm{e}^{-\int_{\tau}^{\tau+\delta} R(t) \mathrm{d} t} \mid R(\tau)=r\right]} .
$$

Under this new measure, it can be proved that the conditional distribution of $R(\tau+$ $\delta$ ) is proportional to a non-central chi-square random variable. For a detailed discussion on the properties of square-root processes, see Jamshidian $(1995,1996)$. More precisely, under $\widetilde{Q}, R(\tau+\delta) / \eta$ has the non-central chi-square distribution with non-centrality parameter $\lambda$ and with $d$ degrees of freedom, where

$$
\lambda=\frac{8 \gamma^{2} \mathrm{e}^{\gamma \delta} r}{\sigma^{2}\left[(\gamma+\kappa)\left(\mathrm{e}^{\gamma \delta}-1\right)+2 \gamma\right]\left(\mathrm{e}^{\gamma \delta}-1\right)},
$$

and where $\gamma, \eta$, and $d$ are defined as in the theorem's statement. Now, we can rewrite $A_{k, i}^{m}$ in terms of the new probability measure $\widetilde{Q}$ as follows:

$$
\begin{aligned}
A_{k, i}^{m} & =E_{m, r_{k}}\left[\mathrm{e}^{-\int_{\tau_{m}}^{\tau_{m+1}} R(t) \mathrm{d} t} I\left(r_{i} \leqslant R\left(\tau_{m+1}\right)<r_{i+1}\right)\right] \\
& =\rho\left(r_{k}, \tau_{m}, \delta\right) \widetilde{Q}\left[r_{i} \leqslant R\left(\tau_{m+1}\right)<r_{i+1}\right] \\
& =\rho\left(r_{k}, \tau_{m}, \delta\right)\left(\Phi_{\lambda_{k}, d}\left(\frac{r_{i+1}}{\eta}\right)-\Phi_{\lambda_{k}, d}\left(\frac{r_{i}}{\eta}\right)\right),
\end{aligned}
$$

where $\rho\left(r_{k}, \tau_{m}, \delta\right)$, the discount factor over the period $\left[\tau_{m}, \tau_{m}+\delta\right]$ when $R\left(\tau_{m}\right)=r_{k}$ is derived using the Laplace transform given by Eq. (18) and $\Phi_{\lambda_{k}, d}$ is the distribution function of a non-central chi-squared with non-centrality parameter $\lambda_{k}$ and $d$ degrees of freedom, with $\lambda_{k}$ defined as in the theorem's statement. The distribution function $\Phi_{\lambda_{k}, d}$ can be written as

$$
\Phi_{\lambda_{k}, d}(z)=\mathrm{e}^{-\lambda_{k} / 2} \sum_{t=0}^{\infty} \frac{\left(\lambda_{k} / 2\right)^{t}}{t !} F_{d+2 t}(z),
$$

where $F_{d+2 t}$ is the chi-square distribution function with $d+2 t$ degrees of freedom.

Combining this result with (21), we obtain

$$
A_{k, i}^{m}=\rho\left(r_{k}, \tau_{m}, \delta\right) \sum_{t=0}^{\infty} \mathrm{e}^{-\lambda_{k} / 2} \frac{\left(\lambda_{k} / 2\right)^{t}}{t !}\left(F_{d+2 t}\left(\frac{r_{i+1}}{\eta}\right)-F_{d+2 t}\left(\frac{r_{i}}{\eta}\right)\right),
$$

where $\lambda_{k}, d$ and $\eta$ are defined as previously. 
With the same change of probability measure, we also obtain

$$
\begin{aligned}
B_{k, i}^{m} & =E_{m, r_{k}}\left[\mathrm{e}^{-\int_{\tau_{m}}^{\tau_{m+1}} R(t) \mathrm{d} t} R\left(\tau_{m+1}\right) I\left(r_{i} \leqslant R\left(\tau_{m+1}\right)<r_{i+1}\right)\right] \\
& =\rho\left(r_{k}, \tau_{m}, \delta\right) \eta E_{m, r_{k}}^{\widetilde{Q}}\left[\frac{R\left(\tau_{m+1}\right)}{\eta} I\left(\frac{r_{i}}{\eta} \leqslant \frac{R\left(\tau_{m+1}\right)}{\eta}<\frac{r_{i+1}}{\eta}\right)\right] .
\end{aligned}
$$

The expectation in this expression can be computed explicitly as

$$
\begin{aligned}
E_{m, r_{k}}^{\widetilde{Q}} & {\left[\frac{R\left(\tau_{m+1}\right)}{\eta} I\left(\frac{r_{i}}{\eta} \leqslant \frac{R\left(\tau_{m+1}\right)}{\eta}<\frac{r_{i+1}}{\eta}\right)\right] } \\
& =\sum_{t=0}^{\infty} \mathrm{e}^{-\lambda_{k} / 2} \frac{\left(\lambda_{k} / 2\right)^{t}}{t !} \int_{r_{i} / \eta}^{r_{i+1} / \eta} y f_{d+2 t}(y) \mathrm{d} y .
\end{aligned}
$$

After integration, it comes that

$$
\begin{aligned}
E_{m, r_{k}}^{\widetilde{Q}} & {\left[\frac{R\left(\tau_{m+1}\right)}{\mu} I\left(\frac{r_{i}}{\mu} \leqslant \frac{R\left(\tau_{m+1}\right)}{\mu}<\frac{r_{i+1}}{\mu}\right)\right] } \\
= & \sum_{t=0}^{\infty} \mathrm{e}^{-\lambda_{k} / 2} \frac{\left(\lambda_{k} / 2\right)^{t}}{t !}\left[-2\left(r_{i+1} f_{d+2 t}\left(\frac{r_{i+1}}{\mu}\right)-r_{i} f_{d+2 t}\left(\frac{r_{i}}{\mu}\right)\right)\right. \\
& \left.+(d+2 t) \times\left(F_{d+2 t}\left(\frac{r_{i+1}}{\mu}\right)-F_{d+2 t}\left(\frac{r_{i}}{\mu}\right)\right)\right] .
\end{aligned}
$$

This implies that

$$
\begin{aligned}
B_{k, i}^{m}= & \rho\left(r_{k}, \tau_{m}, \delta\right) \eta \sum_{t=0}^{\infty} \mathrm{e}^{-\lambda_{k} / 2} \frac{\left(\lambda_{k} / 2\right)^{t}}{t !}\left[-2\left(r_{i+1} f_{d+2 t}\left(\frac{r_{i+1}}{\eta}\right)-r_{i} f_{d+2 t}\left(\frac{r_{i}}{\eta}\right)\right)\right. \\
& \left.+(d+2 t)\left(F_{d+2 t}\left(\frac{r_{i+1}}{\eta}\right)-F_{d+2 t}\left(\frac{r_{i}}{\eta}\right)\right)\right] . \quad \square
\end{aligned}
$$

\section{References}

Ananthanarayanan, A.L., Schwartz, E.S., 1980. Retractable and extendible bonds: the Canadian experience. Journal of Finance 35, 31-47.

Barraquand, J., Martineau, D., 1995. Numerical valuation of high dimensional multivariate American securities. Journal of Financial and Quantitative Analysis 30, 383-405.

Bertsekas, D.P., 1995. Dynamic Programming and Optimal Control, Vol. I and II. Athena Scientific, Belmont, MA.

Black, F., Scholes, M., 1973. The pricing of options and corporate liabilities. Journal of Political Economy 81, 637-654.

Bliss, R.R., Ronn, E.I., 1995. To call or not to call? Optimal call policies for callable U.S. treasury bonds. Economic Review 80, 1-15.

Brennan, M.J., Schwartz, E.S., 1977. Savings bonds, retractable bonds, and callable bonds. Journal of Financial Economics 5, 67-88.

Brennan, M.J., Schwartz, E.S., 1980. Analyzing convertible bonds. Journal of Financial and Quantitative Analysis 15, 907-929. 
Büttler, H.J., Waldvogel, J., 1996. Pricing callable bonds by means of green's function. Mathematical Finance 6, 53-88.

Carverhill, A., 1995. A note on the models of hull and white for pricing options on the term structure. Journal of Fixed Income 5, 89-96.

Chan, K.C., Karolyi, G.A., Longstaff, F.A., Sanders, A.B., 1992. An empirical comparison of alternative models of the short-term interest rate. Journal of Finance 47, 1209-1227.

Courtadon, G., 1982. The pricing of options on default-free bonds. Journal of Financial and Quantitative Analysis 17, 75-100.

Cox, J.C., 1996. The constant elasticity of variance option pricing model. Journal of Portfolio Management 22, 15-17.

Cox, J.C., Ingersoll, J.E., Ross, S.A., 1985a. An intertemporal general equilibrium model of asset prices. Econometrica 53, 363-384.

Cox, J.C., Ingersoll, J.E., Ross, S.A., 1985b. A theory of the term structure of interest rates. Econometrica 53, 385-407.

Dai, Q., Singleton, K., 2000. Specification analysis of affine term structure models. Journal of Finance 55, 1943-1978.

d'Halluin, Y., Forsyth, P.A., Vetzal, K.R., Labahn, G., 2001. A numerical PDE approach for pricing callable bonds. Applied Mathematical Finance 8, 49-77.

Dothan, L.U., 1978. On the term structure of interest rates. Journal of Financial Economics 6, 59-69.

Duffie, D., Kan, R., 1996. A yield-factor model of interest rates. Mathematical Finance 6, 379-406.

Duffie, D., Pan, J., Singleton, K., 2000. Transform analysis and asset pricing for affine jump diffusions. Econometrica 68, 1343-1376.

Duffie, D., Filipovic, D., Schachermayer, W., 2003. Affine processes and applications in finance. Annals of Applied Probability 13, 984-1053.

Elliott, R.J., Kopp, P.E., 1999. Mathematics of Financial Markets. Springer-Verlag, New York.

Feller, W., 1951. Two singular diffusion problems. Annals of Mathematics 54, 173-182.

Geman, H., ElKaroui, N., Rochet, J.C., 1995. Changes of numéraire, changes of probability measure and option pricing. Journal of Applied Probability 32, 443-458.

Hull, J., White, A., 1990a. Pricing interest-rate derivative securities. Review of Financial Studies 3, $573-$ 592.

Hull, J., White, A., 1990b. Valuing derivative securities using the explicit finite difference method. Journal of Financial and Quantitative Analysis 25, 87-100.

Hull, J., White, A., 1993. One factor interest-rate models and the valuation of interest-rate derivative securities. Journal of Financial and Quantitative Analysis 28, 235-254.

Hull, J., White, A., 1994a. Numerical procedures for implementing term structure models I: single-factor models. Journal of Derivatives 2, 7-16.

Hull, J., White, A., 1994b. Numerical procedures for implementing term structure models II: two-factor models. Journal of Derivatives 2, 37-48.

Hull, J., White, A., 1995. A note on the models of hull and white for pricing options on the term structure: response. Journal of Fixed Income 5, 97-103.

Hull, J., White, A., 1996. Using Hull-White interest rate trees. Journal of Derivatives 4, 26-36.

Jamshidian, F., 1989. An exact bond option formula. Journal of Finance 44, 205-209.

Jamshidian, F., 1995. A simple class of square-root interest rate models. Applied Mathematical Finance 2, 61-72.

Jamshidian, F., 1996. Bond futures and option evaluation in the quadratic interest rate model. Applied Mathematical Finance 3, 93-115.

Lamberton, D., Lapeyre, B., 1996. Introduction to Stochastic Calculus Applied to Finance. Chapman \& Hall, London.

Marsh, T.A., Rosenfeld, E.R., 1983. Stochastic processes for interest rates and equilibrium bond prices. Journal of Finance 38, 635-646.

Nelson, C.R., Siegel, A.F., 1987. Parsimonious modeling of yield curves. Journal of Business 60, 473-489.

Øksendal, B., 1995. Stochastic Differential Equations, An Introduction with Applications, fourth ed. Springer, Berlin. 
Protter, P.E., 2004. Stochastic Integration and Differential Equations, second ed. Springer, Berlin.

Rabinovitch, R., 1989. Pricing stock and bond options when the default-free rate is stochastic. Journal of Financial and Quantitative Analysis 24, 447-457.

Richard, S.F., 1978. An arbitrage model of the term structure of interest rates. Journal of Financial Economics 6, 33-57.

Schaefer, S.M., Schwartz, E.S., 1984. A two-factor model of the term structure: an appropriate analytical solution. Journal of Financial and Quantitative Analysis 19, 413-424.

Vasicek, O., 1977. An equilibrium characterization of the term structure. Journal of Financial Economics $5,177-188$ 